\title{
Immunodepression Covid-19-related as a promoting factor for severe Pseudomonas sepsis in an infant with not respiratory symptoms
}

\author{
Letizia Zenzeri, ${ }^{1}$ Antonietta Giannattasio, ${ }^{1}$ Geremia Zito Marinosci, ${ }^{2}$ Eduardo Ponticiello, ${ }^{1}$ \\ Margherita Raffaella Iodice, ${ }^{1}$ Maria Erennia Vitullo, ${ }^{2}$ Vincenzo Tipo ${ }^{1}$ \\ ${ }^{1}$ Pediatric Emergency Unit, Santobono-Pausilipon Children's Hospital; ${ }^{2}$ Pediatric Intensive Care Unit, \\ Santobono-Pausilipon Children's Hospital, Naples, Italy
}

\begin{abstract}
The outbreak of coronavirus disease 2019 caused by severe acute respiratory syndrome coronavirus 2 is a reason of concern worldwide. While a high proportion of adult patients have been severely ill, requiring intensive care assistance and mechanical ventilation, pediatric patients seem to have a less invasive clinical expression of the disease. Reasons for a milder disease in children compared to that seen in adults are yet to be elucidated. Nonetheless, severe and fatal cases have been reported in children and are expected to continue to increase with the growing community transmission and overall current disease prevalence. We report the first case of an infant with a very mild not-respiratory Covid19 infection and a concomitant invasive bacterial sepsis.
\end{abstract}

\section{Introduction}

The first case affected by novel coronavirus disease 2019 (Covid-19) in Italian adult population, has been diagnosed on February 21, 2020. ${ }^{1}$ The worldwide first pediatric case of severe acute respiratory syndrome coronavirus 2 (SARS-CoV-2) infection was detected on January 26, 2020 in China Xiaogan, Hubei province. ${ }^{2}$ In pediatric population, Covid-19 has usually a mild

Correspondence: Zenzeri Letizia, Pediatric Emergency Unit, AORN Santobono-Pausilipon Children's Hospital, via Mario Fiore, 6, Naples, Italy.

Tel.: +39.3293577778

E-mail: zenzeriletizia@gmail.com

Key words: Covid-19; children; sepsis; diarrhea; fever.

Conflict of interest: No one. This work was not supported by any grant.

Ethics approval and consent to participate: The manuscript does not contain any elements that would allow the recognition of the patient.

Received for publication: 13 May 2020.

Accepted for publication: 12 November 2020.

This work is licensed under a Creative Commons Attribution 4.0 License (by-nc 4.0)

C Copyright: the Author(s), 2020

Licensee PAGEPress, Italy

Emergency Care Journal 2020; 16:9105

doi:10.4081/ecj.2020.9105 clinical presentation with heterogeneous and unspecific symptoms. ${ }^{3}$ This in contrast to the high susceptibility of children to some potentially fatal infectious agents.

Lymphopenia is a common laboratory finding in SARS-CoV-2 infection, mainly in severe cases. ${ }^{4}$ In addition, it has been reported significantly lower T cells CD8+ lymphocytes and Natural Killer (NK) cells in patients with severe clinical course compared to those with mild infection and healthy individuals. ${ }^{5}$ These impaired virus-related immunological changes might lead to an increased susceptibility to other, mainly bacterial, agents in severely affected patients. We report the first case of otherwise healthy infant with a very mild Covid-19 infection and a concomitant severe bacterial sepsis.

\section{Case presentation}

A 9 months-old otherwise healthy male infant with a 6-day history of fever and diarrhea, was brought to the Pediatric Emergency Unit of "Santobono-Pausilipon" Children's Hospital. This is the most important pediatric hospital of South Italy and here, many patients are treated and cared; about 100.000 patients under 14 years access each year to our Pediatric Emergency Unit. According to World Health Organization (WHO) recommendations, during this pandemic our hospital ensures that Covid-19 patients can access life-saving treatment, without compromising public health objectives and safety of health worker. ${ }^{6}$ Care for all suspected and confirmed Covid-19 patients is provided in the designated treatment area, according to disease severity and acute care needs.

The studied infant had no respiratory symptoms. Household members had a history of fever lasting 10 days in the last month. Furthermore, father's infant had travelled to one of the first SARSCoV-2 quarantine areas, set up in Northern Italy. No other ill contacts or known contacts with SARS-CoV-2 infected subjects were reported. Based on family history and on the presence of fever, the patient was considered as a suspected Covid-19 case and was diverted to the designated Covid-19 track.

On admission, the infant had very poor general condition and irritability. The state of consciousness was normal. Physical examination revealed a body temperature of $39.5^{\circ} \mathrm{C}$, slight skin mottling, mild dehydration (refill of 3 second), no tachypnoea (respiratory rate was 42 breaths per minute), oxygen saturation of $98 \%$ on room air, clear lungs auscultation and slightly tender abdomen. According to our standard protocol of care for Covid-19, the patient underwent to blood and microbiological exams including blood specimens for cultures and nasopharyngeal swab specimen for 2019-nCoV nucleic acid (by a real-time reverse-transcriptase polymerase-chain-reaction -RT-PCR- assay). Laboratory results are showed in Table 1. We observed a severe decrease of leukocytes and neutrophils and high values of inflammatory markers. 
Infant's serology revealed the presence of $\operatorname{IgM}$ and $\operatorname{IgG}$ for Covid19 infection, while mother's serology (both $\operatorname{IgM}$ and $\operatorname{IgG}$ ) was negative. Covid-19 was confirmed by RT-PCR on nasopharyngeal swab specimen. Chest X-ray revealed no pathologic findings. Considering the compromised general conditions and laboratory results, our infant also underwent diagnostic evaluation for invasive bacterial disease. This evaluation included urine exam, blood, stool and Cerebro-Spinal Fluid (CSF) cultures, followed by empiric antibiotic therapy. No signs of urinary infection were found; CSF was clear, with normal leucocytes count and glucose level, and slightly decrease of protein $(14 / \mathrm{mg} / \mathrm{dl})$. SARS-CoV-2 realtime RT-PCR and culture on CSF were negative. Coproculture was negative. Immunological investigation was also performed. TBNK lymphocyte subset analysis revealed decreased percentage of CD4+ $(23 \%$, normal value $44 \pm 11)$ and CD $8+(7 \%$, normal value $21 \pm 9)$ cells and slightly low NK cells $(6 \%$, normal value $10 \pm 3)$. Cytokine detection assay revealed increased levels of interleukin6 (IL-6), (25,9 pg/mL, normal value $0-5 \mathrm{pg} / \mathrm{mL})$.

Empiric treatment with parental cephalosporin (ceftazidime) was started. Considering the poor general conditions, he was transferred to our Sub-intensive Pediatric Unit, and was treated according to pediatric sepsis guidelines. ${ }^{7}$ Here, despite the initial clinical presentation, patient conditions were stable. Patient showed persistent fever, abdominal pain and a certain degree of irritability. Head CT scan was normal, as well as ultrasound scan of the abdomen. There were no signs of pulmonary dysfunction, beside a mild degree of tachypnoea that resolved when fever broke down; blood gas analysis was normal and the patient had no needs of oxygen therapy or ventilatory assistance. Laboratory tests were then repeated after 24 hours (Table 1). Blood culture resulted positive for Pseudomonas aeruginosa susceptible to cephalosporin. No antiviral medication was used. The patient was transferred to the Pediatric Infectious Unit to continue antibiotic and supportive therapy without need to new admission in intensive care ward.

\section{Discussion}

Pediatric patients with Covid-19 have their own clinical features and different therapeutic response compared with adult infected population. ${ }^{8}$ Nausea and vomiting have been frequently observed in pediatric cohort, while fever, present in almost all infected adults, may be absent in a percentage of children, even in presence of a severe clinical picture. 3,9 However, respiratory involvement is reported in almost all severe cases of pediatric Covid-19 infection. ${ }^{9,10}$ Despite the absence of respiratory involvement, our patient was considered as a suspected Covid-19 case because of presence of fever. Considering the mild onset of Covid19 disease in the majority of pediatric patients, the severe clinical picture of our infant led to search for etiologies other than SARS$\mathrm{CoV}-2$ and to perform the workup of potential invasive bacterial disease. Moreover, in Covid-19 patients, C-reactive protein and procalcitonin levels are often normal and a level of procalcitonin $>0.5 \mathrm{ng} / \mathrm{mL}$ usually suggests a bacterial co-infection. ${ }^{11}$ In our case, clinical improvement with empiric antibiotic therapy and laboratory results at baseline strongly suggested a bacterial etiology, subsequently confirmed by blood culture.

It has been reported that the essence of severe Covid-19 is a sepsis induced by viral infection. ${ }^{12}$ Unfavourable progression, combined with a high incidence of sepsis, strongly suggest the involvement of significant changes in the host's immune response during SARS-CoV-2 infection. It is reasonable to assess that in our case the compromised clinical condition and the laboratory exams were not related to Covid-19 infection itself but to a bacterial coinfection. However, while in adult cases with an unfavourable course SARS-CoV-2 infection itself presented with a severe respiratory involvement, our patient had a clinical silent Covid-19 infection. Covid-19 can induce an aberrant non-effective host immune response associated with severe lung involvement. ${ }^{9} \mathrm{~T}$ cells play a critical role in antiviral immunity. It has been reported that $\mathrm{T}$ cell counts are significantly reduced in Covid-19 patients, and the surviving $\mathrm{T}$ cells appear functionally exhausted. ${ }^{4}$ Low $\mathrm{T}$ cell count might be predictive of further clinical deterioration; as a consequence, early and more aggressive therapeutic interventions may be required in these patients. Our patient underwent a decreased in total $\mathrm{T}$ cells, CD4+ T and NK cells, and mostly importantly in $\mathrm{CD} 8+\mathrm{T}$ cells. Cytokine storm is a well-known phenomenon in respiratory viral infections. In SARS-Cov-2 adults increased plasma concentrations of pro-inflammatory cytokines has been found. ${ }^{4,13}$ In our patient, we found an increased secretion of IL-6. This

Table 1. Patient laboratory results per hour since admission.

\begin{tabular}{|c|c|c|c|}
\hline Test & & $\begin{array}{l}\text { Time (hours): } \\
\text { Admission }\end{array}$ & 12 \\
\hline $\mathrm{CBC}$ & 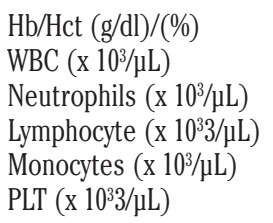 & $\begin{array}{ll}9.0 / 26.10 & \\
1.84 & \\
0.48 & \\
0.81 & \\
0.62 & \\
336 & 266\end{array}$ & $\begin{array}{l}8.0 / 23.30 \\
0.91 \\
0.31 \\
0.45 \\
0.15\end{array}$ \\
\hline CMP & $\begin{array}{l}\text { BUN/Cr (mg/dl) /(g/dl) } \\
\text { AST/ALT (units/L) }\end{array}$ & $\begin{array}{l}16 / 0.23 \\
25 / 42\end{array}$ & $\begin{array}{l}19 / 0.21 \\
\operatorname{lug}-26\end{array}$ \\
\hline Inflammatory markers & $\begin{array}{l}\text { PCT (ng/mL) } \\
\text { CRP (mg/L) } \\
\text { Ferritin(ng/mL) } \\
\text { Albumin (g/dL) } \\
\text { D-dimero (ng/mL) }\end{array}$ & $\begin{array}{l}61.34 \\
412.76 \\
686.00 \\
3.0 \\
1300.00\end{array}$ & $\begin{array}{l}38.60 \\
338.06 \\
785.50 \\
2.8\end{array}$ \\
\hline
\end{tabular}

CBC: Complete Blood Cell count; Hb: Hemoglobin; Hct: Hematocrit; WBC: White Blood Cells; PLT: platelets; CMP: Comprehensive Metabolic Panel; BUN: Blood Urea Nitrogen test (n.v. 10-38 mg/dL); Cr: creatinine (n.v. 0.10-0.40 mg/dL); AST: aspartate aminotransferase (n.v. 5-58 U/L; ALT: alanine aminotransferase (n.v. 8-40 U/L); PCT: procalcitonin (n.v.: 0.0- 0.5 ng/mL); CRP: C-Reactive Protein (n.v. 0.0-5.0mg/L); Ferritin (n.v. 10.00$320.00 \mathrm{ng} / \mathrm{mL}$ ); Albumin (n.v. $3.4-4.8 \mathrm{~g} / \mathrm{dL}$ ); D-dimero (n.v. 0.00-270.00 ng/mL) 
cytokine plays a pathological role in acute and chronic inflammation and infection. ${ }^{14}$ Furthermore, IL- 6 causes compromised tissue repair by shifting acute inflammation into a more chronic profibrotic state through induction of Th1 cell responses as a consequence of recurrent inflammation. ${ }^{15}$

It is possible to hypothesize that these Covid-19 induced changes in immune response might have led to an increased susceptibility to develop a bacterial infection in our otherwise healthy infant. As for the specific etiology, sepsis due to Pseudomonas aeruginosa commonly is usually hospital-acquired and affects children with underlying clinical conditions. On the other hand, although the gastrointestinal tract is not the common infection site of Pseudomonas aeruginosa, it has been reported that it can be a causative agent for diarrhea in children without pre-existing disease. ${ }^{16}$ The impaired host immune response Covid-19 induced could have promoted translocation of Pseudomonas aeruginosa in bloodstream.

In literature, few cases of infants with Covid-19 infection and clinical signs of sepsis have been reported but in none of them a confirmed bacterial etiology of sepsis has been found. ${ }^{17,18}$

\section{Conclusions}

In children with non-specific symptoms of Covid infection, with a familial history of fever and/or respiratory involvement and/or epidemiological warning factors, performance of PCR testing for Covid-19 it is mandatory. Furthermore, in our experience, the effects related to Covid-19 infection on the immune system as a risk factor in promoting bacterial infection appears to be the key message for those working in Emergency departments. Therefore, in the presence of a septic appearance and elevated inflammatory indexes of a patient, searching for other aetiologies remains fundamental in clinical practice.

Finally, this case highlights a likelihood of poor performance of current case definitions used to contain Covid-19, mainly in infants. There is an urgent need to better understand the full clinical spectrum of Covid-19 in the pediatric population in order to refine public health containment strategies.

\section{References}

1. Remuzzi A, Remuzzi G. Covid-19 and Italy: what next? Lancet 2020;395:1225-8.

2. Zhang YH, Lin DJ, Xiao MF, et al. [2019 novel coronavirus infection in a three-month-old baby]. Zhonghua Er Ke Za Zhi 2020;58:182-4. [Article in Chinese].

3. Yonker LM, Neilan AM, Bartsch Y, et al. Pediatric Severe Acute Respiratory Syndrome Coronavirus 2 (SARS-CoV-2): Clinical Presentation, Infectivity, and Immune Responses. J
Pediatr 2020;227:45-52.E5

4. Diao B, Wang C, Tan Y, et al. Reduction and Functional Exhaustion of T Cells in Patients with Coronavirus Disease 2019 (Covid-19). Front Immunol 2020;11:827.

5. Yaqinuddin A, Kashir J. Innate immunity in Covid-19 patients mediated by NKG2A receptors, and potential treatment using Monalizumab, Cholroquine, and antiviral agents. Med Hypotheses 2020;140:109777.

6. WHO. Updated WHO recommendations for international traffic in relation to COVID-19 outbreak. Available at: https://www.who.int/news-room/articles-detail/updated-whorecommendations-for-international-traffic-in-relation-tocovid-19-outbreak

7. Weiss SL, Peters MJ, Alhazzani W, et al. Surviving Sepsis Campaign International Guidelines for the Management of Septic Shock and Sepsis-Associated Organ Dysfunction in Children. Pediatr Crit Care Med 2020;21:e52-e106.

8. Chen ZM, Fu JF, Shu Q. New Coronavirus: new Challenges for Pediatricians. World J Pediatr 2020;16:222.

9. Sun D, Li H, Lu X, et al. Clinical features of severe pediatric patients with coronavirus disease 2019 in Wuhan: a single center's observational study. World J Pediatr 2020;16:251-9.

10. Cai JH, Wang XS, Ge YL, et al. [First case of 2019 novel coronavirus infection in children in Shanghai]. Zhonghua Er Ke Za Zhi 2020;58:E002. [Article in Chinese].

11. Cai J, Xu J, Lin D, et al. A Case Series of children with 2019 novel coronavirus infection: clinical and epidemiological features. Clin Infect Dis 2020;71:1547-51.

12. Li H, Liu L, Zhang D, et al. SARS-CoV-2 and viral sepsis: observations and hypotheses. Lancet 2020;395:1517-20.

13. Huang C, Wang Y, Li X, et al. Clinical features of patients infected with 2019 novel coronavirus in Wuhan, China. Lancet 2020;395:497-506.

14. Jones SA, Jenkins BJ. Recent insights into targeting the IL-6 cytokine family in inflammatory diseases and cancer. Nat Rev Immunol 2018;18:773-89.

15. Fielding CA, Jones GW, McLoughlin RM, et al. Interleukin-6 signaling drives fibrosis in unresolved inflammation. Immunity 2014;40:40-50.

16. Chuang CH, Janapatla RP, Wang YH, et al. Pseudomonas aeruginosa-associated Diarrheal Diseases in Children. Pediatr Infect Dis J 2017;36:1119-23.

17. Coronado Munoz A, Nawaratne U, McMann D, et al. LateOnset Neonatal Sepsis in a Patient with Covid-19. N Engl J Med 2020;382:e49.

18. Kamali Aghdam M, Jafari N, Eftekhari K. Novel coronavirus in a 15-day-old neonate with clinical signs of sepsis, a case report. Infect Dis (Lond) 2020;52:427-9. 